\title{
Methods for fabricating arrays of holes using interference lithography
}

\author{
A. Fernandez, J. Y. Decker, S. M. Herman, \\ D. W. Phillion, D. W. Sweeney, M. D. Perry \\ Lawrence Livermore National Laboratory \\ Livermore, CA 94551
}

This paper was prepared for submittal to the 41 st International Conference on Electron, Ion and Photon Beam Technology and Nanofabrication May 27-30, 1997

Dana Point, CA

May 28, 1997

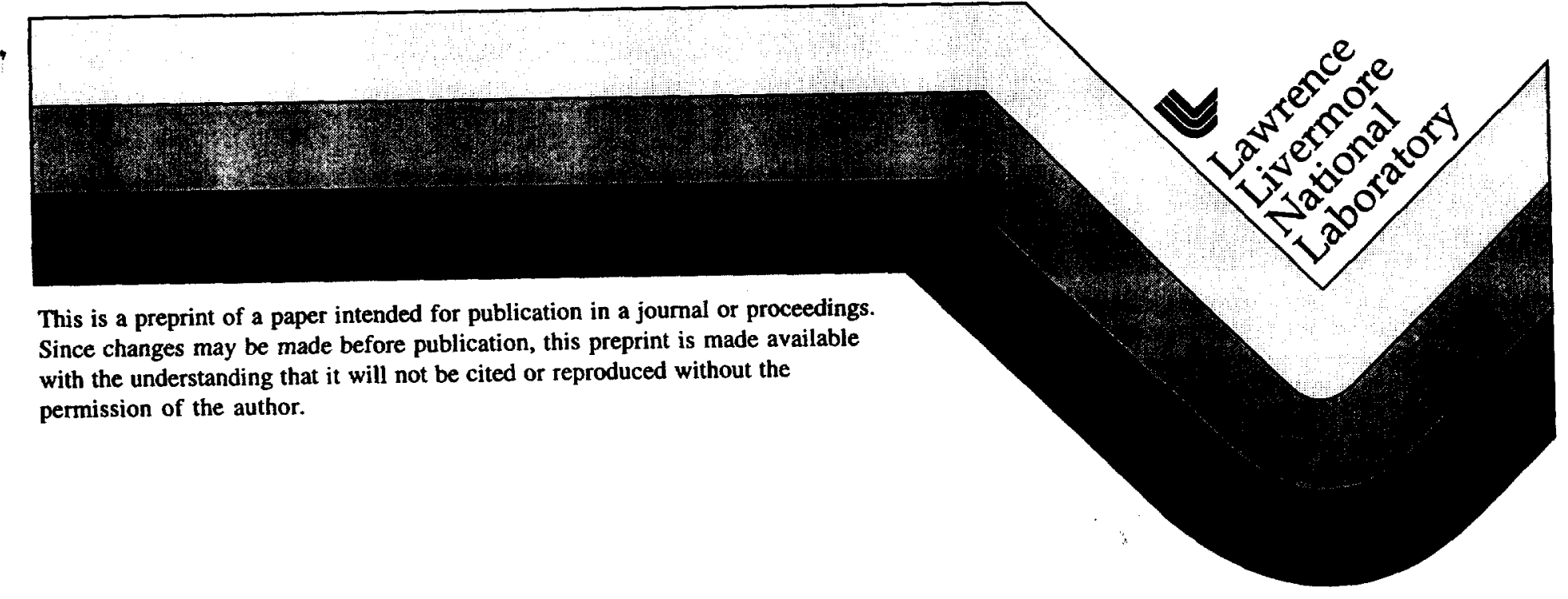




\section{DISCLAIMER}

This document was prepared as an account of work sponsored by an agency of the United States Government. Neither the United States Government nor the University of California nor any of their employees, makes any warranty, express or implied, or assumes any legal liability or responsibility for the accuracy, completeness, or usefulness of any information, apparatus, product, or process disclosed, or represents that its use would not infringe privately owned rights. Reference herein to any specific commercial products, process, or service by trade name, trademark, manufacturer, or otherwise, does not necessarily constitute or imply its endorsement, recommendation, or favoring by the United States Government or the University of California. The views and opinions of authors expressed herein do not necessarily state or reflect those of the United States Government or the University of California, and shall not be used for advertising or product endorsement purposes. 


\section{DISCLAIMER}

This document was prepared as an account of work sponsored by an agency of the United States Government. Neither the United States Government nor the University of California nor any of their employees, makes any warranty, express or implied, or assumes any legal liability or responsibility for the accuracy, completeness, or usefulness of any information, apparatus, product, or process disclosed, or represents that its use would not infringe privately owned rights. Reference herein to any specific commercial products, process, or service by trade name, trademark, manufacturer, or otherwise, does not necessarily constitute or imply its endorsement, recommendation, or favoring by the United States Government or the University of California. The views and opinions of authors expressed herein do not necessarily state or reflect those of the United States Government or the University of California, and shall not be used for advertising or product endorsenient purposes. 


\title{
Methods for fabricating arrays of holes using interference lithography
}

\author{
A. Fernandez, J. Y. Decker, S. M. Herman, D. W. Phillion, \\ D. W. Sweeney and M. D. Perry \\ Lawrence Livermore National Laboratory, Livermore, CA 94551
}

\begin{abstract}
Optical interference lithography offers a robust patterning technology capable of achieving high spatial resolution over extremely large field sizes $(\sim 1 \mathrm{~m})$. Here, we compare two different approaches for fabricating arrays of holes using interferometric techniques. We show that by applying an image reversal process to standard two-beam interference lithography, arrays of high aspect ratio holes can be generated. This process scales well to submicron periods and allows holes as small as $0.1 \mu \mathrm{m}$ to be patterned. Next, we present an analysis of the multiple-beam approach for patterning holes. We demonstrate that while the formation of higher contrast intensity patterns is possible by interfering four or more beams, the shape and modulation depth of such patterns are inherently sensitive to relative phase variations. This dependence complicates the application of multiple-beam techniques for the patterning large uniform arrays of resist structures.
\end{abstract}




\section{Introduction}

Optical interference lithography has been successfully applied for well over a decade to manufacture periodic structures for a wide variety of uses. The simplest embodiment of this technique is to interfere two coherent wavefronts to form a sinusoidal intensity pattern in space. By exposing a photosensitive material to the this pattern, a 1D surface relief or volumetric grating can be generated. Two-dimensional gratings can be produced by overlaying two sequential exposures at $90^{\circ}$. The great advantage of utilizing such a simple method to form an image is that deep submicron resolution can be achieved with an effectively infinite depth of field. Moreover, this resolution can be obtained over field sizes that approach $1 \mathrm{~m}$. Recently, interest in interference lithography has grown as new, commercially viable applications have emerged. In particular, interference lithography is an attractive technology for patterning field emission flat panel displays..$^{1-4}$ Interference lithography also shows potential for defining the critical features on future generation integrated circuits, 5 and shows promise as a means of patterning high-density storage media based on single-domain magnetic particles. ${ }^{6}$

The use of interference lithography for applications that are potentially high-volume will depend on the ultimate limits of uniformity, throughput, process control, and repeatability that can be achieved. Demonstrating that a wide range in resist profiles can be obtained is also critically important. A recent study has shown that square arrays of sub-quarter micron, high-aspect-ratio posts (3:1) can readily be patterned using the crossed exposure technique and high-contrast $\mathrm{i}$-line resists. ${ }^{4}$ Fabricating arrays of high aspect ratio holes, which have particular application to field emission displays, using the same exposure process has proven to be more challenging. Compared to posts, holes are patterned with shallower sidewall slopes and significantly narrower process windows.3,4 This limitation arises because 
contrast in the 2D dose distribution is relatively poor near the maxima, where holes are patterned, due to the sinusoidal shape of the interference pattern and to the incoherent nature of the crossed exposure process. There are several strategies for overcoming this handicap. One approach is to reverse the tonality of the process so that holes are formed at the minima of the dose distribution, where contrast is considerably higher. Two methods can be employed, one of which is to use negative resists. In the optical wavelength range, however, negative resists typically suffer from lower photosensitivity and poorer resolution compared to positive resists. An alternative method is to use image reversal. Image reversal processes can achieve high resolution and can actually improve overall resist performance. ${ }^{7}$

Another intriguing approach for fabricating arrays of holes is to modify the shape of the incident intensity distribution by interfering more than two beams. The coherent addition of multiple beams can produce an intensity profile that is much sharper than a simple two-beam pattern. ${ }^{3,5}$ A positive tone process can then be used to produce arrays of holes. Although many different configurations of multiple beams can increase image contrast, only those which satisfy certain symmetry requirements preserve the large depth of field characteristic of a two-beam pattern. Chen et al. in a recent survey of the multiple-beam patterns identified the four-beam arrangement-whereby four equal intensity beams converge at equal angles along two orthogonal planes-as one such configuration. ${ }^{3}$ They demonstrated the potential for multiple-beam techniques by patterning sparse holes arrays, although they did not address issues such as uniformity and process control.

In this study, a comparison of two different methods for fabricating arrays of submicron holes using interference lithography is presented. We first discuss experimental results of applying image reversal to standard two-beam interference lithography. We describe how this process scales to submicron periods and assess the viability of this process for large scale production of hole arrays. Next, we consider 
the multiple-beam approach for printing resist structures. We show that multiple-beam interference patterns are complicated by an inherent sensitivity to the relative phases of the beams when four or more beams are used. We discuss the impact of this sensitivity on critical issues such as uniformity and reproducibility in the context of developing a production worthy process.

\section{Image Reversal Method for Patterning Arrays of Holes}

Combining image reversal with interference lithography offers the prospect of patterning complementary structures to those that can readily be generated using a positive tone process. Image reversal has long been recognized as a means of reversing the tone of diazonaphthoquinone-based resists. Moreover, it can enhance resist performance by increasing resist contrast and by reducing process sensitivity to reflective substrates or difficult topography. ${ }^{7}$

\section{A. Fabrication process}

Two interference lithography systems are used in this study. One system utilizes a $1 \mathrm{~W} \mathrm{Kr}$ ion laser operating at $413 \mathrm{~nm}$ and the other utilizes a $1 \mathrm{~W}$ Ar ion laser operating at $351 \mathrm{~nm}$. (The details of the $\mathrm{Kr}$ laser system and the Ar laser system are discussed in Ref. ${ }^{4}$ and Ref. ${ }^{2}$, respectively.) An interference pattern is formed by splitting the laser beam, expanding the split beams through spatial filters, and then recombining the two beams at the exposure plane. The period of the interference pattern is controlled by adjusting the intersection angle. In both systems, the laser outputs are adjusted for $\mathrm{TEM}_{00}$ operation and active fringe stabilization is used.

Two-dimensional arrays of resist structures are produced by the following procedure. Resist coated Si wafers are exposed to the interference pattern once, then rotated $90^{\circ}$, and exposed again. (An anti-reflective coating is used to suppress standing waves.) The wafers are then split in half. One half of each substrate is baked in an ammonia filled oven (600 Torr) at a nominal temperature of $90^{\circ} \mathrm{C}$ for between 
30 and $40 \mathrm{~min}$. This post-bake step promotes the formation of an insoluble matrix in the exposed regions, ${ }^{8}$ and is followed by a flood exposure with a UV light source $\left(150 \mathrm{~mJ} / \mathrm{cm}^{2}\right)$ and then development. The other half of each wafer is directly developed after the interference exposures, so that image reversal can be compared to the positive process.

\section{B. High aspect ratio hole arrays}

Just as arrays of high aspect ratio resist posts can be fabricated using the positive process, arrays of high aspect ratio holes can be produced using image reversal. Fig. 1(a) shows a square array of holes centered on a $1 \mu \mathrm{m}$ grid produced by image reversal. The holes are $0.32 \mu \mathrm{m}$ in diameter, as measured diagonally relative to the square grid, and are $0.65 \mu \mathrm{m}$ deep, giving an aspect ratio of 2:1. The edges of the holes are sharp and well defined. A cross-sectional view of a sample exposed at a higher dose is shown in Fig. 1(b) and reveals that the sidewalls of the holes are nearly vertical. The sharpness of the profiles is indicative of the high contrast attainable with this approach.

For a set interference period, the array structure is controlled by varying the exposure dose. ${ }^{4}$ For the positive tone process, holes are formed at low doses where the maxima of the two interference exposures overlap. At higher doses, posts are formed where the minima overlap. For the image reversal process, the exact opposite sequence of resist structures is produced. Structure width measurements for both the positive process and image reversal are plotted in Fig. 2 for a series of $1 \mu \mathrm{m}$ period exposures. The two curves feature a similar shape over the entire dose range, which indicates nearly identical process latitudes for the two processes. In addition, the threshold dose at which the array structure inverts from one type of structure (holes or posts) to the other type is the same for both processes, as indicated in the graph. The only difference between the two curves is that there is an 
approximately constant bias between them. At a fixed exposure dose, the image reversal process results in a larger structure than the positive process. We find that the magnitude of the bias depends on processing parameters, and can be reduced by using a higher post-bake temperature of $100^{\circ} \mathrm{C}$ or a lower flood exposure dose of $100 \mathrm{~mJ} / \mathrm{cm}^{2}$.

A detailed study of applying image reversal at submicron interference periods reveals that this process scales remarkably well to smaller dimensions. ${ }^{9}$ Shown in Fig. 3 is an array of $0.21 \mu \mathrm{m}$ diameter holes centered on a $0.35 \mu \mathrm{m}$ grid. The aspect ratio of these holes is 2:1, and the resist slope is close to vertical. Similar to the holes fabricated with larger interference periods, the process latitude for patterning holes with image reversal is nearly identical to the latitude for patterning dots with the positive process. Together, these results demonstrate the high resolution capability of this technique and the potential for developing a robust manufacturing process.

\section{Multiple Beam Interference Patterns}

Another method of producing arrays of holes interferometrically is to use multiple beams to form the interference pattern. The main advantage is that higher contrast images can be produced, thus allowing one to use a standard positive tone process. ${ }^{3}$ In conventional optical lithography systems, an increase in image contrast is typically gained at the expense of a reduction in the depth of focus. The corresponding trade-off in multiple-beam interferometric lithography is an increased sensitivity to phase variations. In particular, the shape and modulation depth of a multiple-beam interference pattern can strongly depend on the relative phases of the beams when the number of interfering beams is four or greater. 


\section{A. Four-beam pattern}

To illustrate the phase dependence of multiple-beam interference patterns, we analyze the interference pattern formed by intersecting four coherent plane waves, taking into account the possibility for phase changes. We consider the four-beam configuration in which four equal intensity wavefronts converge at equal angles along two orthogonal planes-two plane waves incident at $\pm \theta$ on one plane and two plane waves incident at $\pm \theta$ on an orthogonal plane. The intensity contrast in the interference pattern is maximized when one pair of plane waves is TE and the other pair is TM. The intensity distribution is found by taking the square of the sum of the electric field vectors and averaging this quantity over time. The result is the expression ${ }^{10}$

$$
\begin{aligned}
\mathrm{I}(x, y)= & E_{0}{ }^{2}\left(2+\cos 2 \theta \cos (2 k x \sin \theta)+\cos \left(2 k y \sin \theta+\phi_{0}\right)\right. \\
& \left.+2 \cos \theta \cos (k x \sin \theta)\left[\cos (k y \sin \theta)+\cos \left(k y \sin \theta+\phi_{0}\right)\right]\right\}
\end{aligned}
$$

where $E_{0}$ is the amplitude of each plane wave, and $k$ is the magnitude of the propagation vector. The quantity $\phi_{0}$ which we refer to as the net relative phase, is related to the phase constants of the four incident beams by the expression $\phi_{0}=\left(\phi_{1}+\phi_{2}\right)-\left(\phi_{3}+\phi_{4}\right)$, where $\phi_{1}$ and $\phi_{2}$ are the phase constants of the TE beams, and $\phi_{3}$ and $\phi_{4}$ are the phase constants of the TM beams.

An important property of a two-beam interference pattern is that phase shifts only result in a translation of the profile. The shape and modulation depth of the pattern are invariant to these changes. This is not true of the four-beam interference pattern. As indicated by Eq. (1), the four-beam intensity profile is a periodic function of the net relative phase $\phi_{0}$. If the net relative phase is even multiple of $\pi$, a perfectly modulated, high-contrast intensity distribution is produced, as displayed in Fig. 4(a). However, if the net relative phase is shifted by $\pi$ from this condition, a pattern with reduced modulation and significantly weaker contrast is formed, as shown in 
Fig. 4(b). While the high-contrast pattern in Fig. 4(a) is well suited for printing hole arrays, the low-contrast pattern in Fig. 4(b) is not. In fact, the contrast in the out-of-phase pattern is actually weaker than it is for a crossed two-beam exposure.

The phase dependence exhibited by the four-beam pattern is an intrinsic property that generally applies to all interference patterns constructed from four or more coherent plane waves. ${ }^{10}$ The basic problem is to locate the points on a given plane (the exposure plane) where all the interfering plane waves satisfy some fixed set of relative phase conditions (such as where they are all in phase). This becomes an over constrained problem if the number of plane waves exceeds three. In this case, there are at least three relative phase conditions that need to be satisfied, but there are only two independent space variables on any given plane. Consequently, for an arbitrary set of phase constants $\left(\phi_{1}, \phi_{2}, \ldots \phi_{n}\right)$, there may be no points on the plane where the phase conditions are met when $n \geq 4$. The corollary to this statement is that a solution may exist for specific sets of phase constants. Hence a sensitivity to phase will exist if the number of beams is four or greater.

\section{B. Considerations for lithography}

The inherent sensitivity to phase variations of multiple-beam interference patterns complicates their practical application for several reasons. Consider the four-beam pattern discussed above. In order to obtain an interference pattern with optimized modulation and contrast (the in-phase pattern), the phase of at least one beam would need to be precisely tuned. For reproducible exposures, these adjustments would need to be made continuously to compensate for environmental disturbances to the system, which can cause optical path lengths to drift. A control system would need some means of directly monitoring the pattern and a way of discriminating between different relative phase conditions. Realizing such a control scheme may become quite challenging for interference periods of 
$1 \mu \mathrm{m}$ and smaller. Other control schemes based on a more indirect mechanism can be envisioned, but these systems would require frequent calibration.

Uniformity is another critical issue that becomes more difficult to maintain when multiple-beam interference patterns are employed. Equation (1), which describes a uniform intensity pattern in the $x-y$ plane, only applies for perfectly aligned beams. If the incident angle of any beam deviates from the nominal angle of $\theta$, a phase mismatch error will be introduced, relative to the other beams, that grows in magnitude away from any in-phase point. As a result, a more complex 3D moiré pattern will be formed. On any plane parallel to the $x-y$ plane, the shape of the intensity pattern will vary periodically from the in-phase pattern [Fig. 4(a)] to the out-of-phase pattern [Fig. 4(b)] in the direction of the tilt. For a small alignment error of $\delta$, the distance between adjacent out-of-phase points is $\sim \lambda /(\delta \cos \theta)$ along the direction of tilt.

The implication of the above argument is that that a tolerance must be placed on the alignment and collimation of the beams to ensure a uniformly modulated intensity pattern across the incident plane. The larger the field size of interest is, the tighter the tolerance must be. If we set the criterion that the distance between out-of-phase points to be 10 times the field size, the amount of tilt error that can be tolerated for a given field size can be estimated. For a field size of $2 \mathrm{~cm}$ (typical of semiconductor integrated circuits), a wavelength of $365 \mathrm{~nm}$ and an incident angle of $30^{\circ}$, an angular accuracy of $4 \mu \mathrm{rad}$ would be required. For a field size of $50 \mathrm{~cm}$ (typical for flat panel displays), an accuracy of $0.3 \mu \mathrm{rad}$ would be necessary.

\section{Summary}

The results we have presented on the image reversal method demonstrate the high resolution capability of this technique and the potential for utilizing this approach to produce uniform arrays of sub-quarter micron holes over large areas. 
The main benefits of this method arise from the fact that it allows the use of standard two-beam interference lithography for generating the latent image in the resist. This greatly simplifies the imaging part of the process in comparison to multiple-beam techniques. Two-beam interference patterns do not depend on relative phases (other than simple translations of the pattern) and do not require tight tolerances on beam alignment and collimation to ensure uniformity. Furthermore, the cost-savings of assembling a two-beam system over a multiple-beam system may be quite substantial for applications that do not require precise placement of the resist pattern. In this case, the need for beam collimation would be relaxed for the two-beam system but not for the multiple-beam system.

The obvious disadvantage of the image reversal method for fabricating hole arrays is an increase in process complexity over a positive tone process. In addition, the exposure doses required for image reversal may be larger, and this would translate into lower throughput. In the long run, therefore, it may be more cost-effective to overcome the technical challenges associated with multiple-beams than to rely on complex processing schemes, just as the semiconductor industry has always opted for newer imaging technology than rely on advanced processing techniques such as image reversal.

This work was performed at the Lawrence Livermore National Laboratory for the US Department of Energy under contract No. W-7405-Eng-48.

\section{References}

${ }^{1}$ C. O. Bozler, C. T. Harris, S. Rabe, D. D. Rathman, M. A. Hollis, and H. I. Smith, J. Vac. Sci. Technol. B 12, 629 (1994).

2 J. P. Spallas, A. M. Hawryluk, and D. R. Kania, J. Vac. Sci. Technol. B 13, 1973 (1995). 
3 X. Chen, S. H. Zaidi, S. R. J. Brueck, and D. J. Devine, J. Vac. Sci. Technol. B 14, 3339 (1996).

${ }^{4}$ A. Fernandez, H. T. Nguyen, J. A. Britten, R. D. Boyd, D. R. Kania, A. M. Hawryluk, and M. D. Perry, to appear in J. Vac. Sci and Technol. B , May/June (1997).

5 S. H. Zaidi, and S. R. J. Brueck, J. Vac. Sci. Technol. B 11, 658 (1993).

${ }^{6}$ A. Fernandez, P. J. Bedrossian, S. L. Baker, S. P. Vernon, and D. R. Kania, IEEE Trans. Magn. 32, 4472 (1996).

7 R. Dammel, Diazonaphthoquinone-based Resists, (SPIE, Bellingham, WA, 1993).

${ }^{8}$ H. Klose, R. Siguish, and W. Arden, IEEE Trans. Electron Devices 32, 1654 (1985).

9 J. Y. Decker, A. Fernandez, and D. W. Sweeney, submitted toJ. Vac. Sci. Technol. B, (1997).

10 A. Fernandez, and D. W. Phillion, submitted toJournal of Applied Optics , (1997). 


\section{Figure Captions}

Fig. 1 SEM micrographs of arrays of holes on a $1 \mu \mathrm{m}$ pitch produced by overlying two sequential two-beam interference exposures at $90^{\circ}$ and then applying image reversal. A $413 \mathrm{~nm} \mathrm{Kr}$ ion laser was used. (a) An overhead view of sample that received a total exposure dose of $180 \mathrm{~mJ} / \mathrm{cm}^{2}$. The holes are $0.32 \mu \mathrm{m}$ in diameter, as measured diagonally across the square grid, and are $0.65 \mu \mathrm{m}$ deep, for an aspect ratio of 2:1. (b) Cross-sectional view, taken parallel to the grid, of a sample that received a total dose of $210 \mathrm{~mJ} / \mathrm{cm}^{2}$.

Fig. 2 A plot of resist structure width vs. exposure dose for 2D arrays of structures patterned by the crossed exposure technique. Data for both the image reversal process and the positive process are displayed. A $413 \mathrm{~nm} \mathrm{Kr}$ ion laser was used, and the interference period was $1 \mu \mathrm{m}$. The structures were measured diagonally with respect to the interference fringes.

Fig. 3 SEM of an array of holes on a $0.35 \mu \mathrm{m}$ pitch produced by the crossed exposure technique and image reversal. A $351 \mathrm{~nm}$ Ar ion laser was used, and the total exposure dose was $65 \mathrm{~mJ} / \mathrm{cm}^{2}$. The holes are $0.21 \mu \mathrm{m}$ in diameter and are $0.42 \mu \mathrm{m}$ deep, for an aspect ratio of 2:1. Arrays produced at this length scale demonstrate that this method for producing holes scales well to submicron periods.

Fig. 4 The $2 \mathrm{D}$ intensity distribution of a four-beam interference pattern $\left(\theta=30^{\circ}\right)$ under different relative phase conditions. For these patterns, the TE plane waves are incident from the $\pm y$ directions and the $T M$ plane waves are incident from the $\pm x$ directions. The high contrast pattern in (a) occurs when the net relative phase $\phi_{0}$ is an even multiple of $\pi$. The the low-contrast pattern in (b) occurs when $\phi_{0}$ is an odd multiple of $\pi$. The grey scales are normalized to the maximum intensity in (a), and the spatial scales are normalized to $k=1$. 


\section{A. Fernandez et. al}
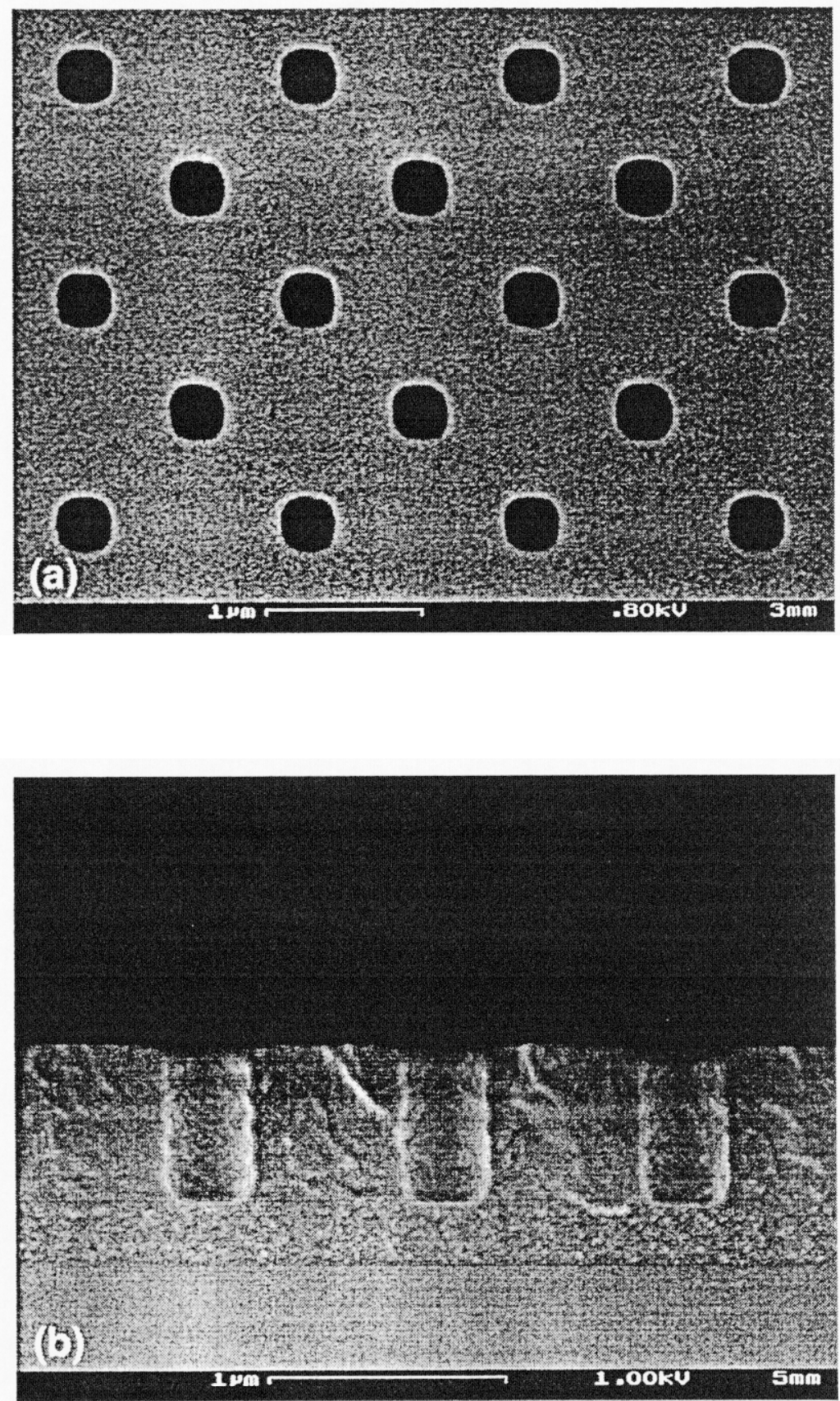

Figure 1 
A. Fernandez et. al

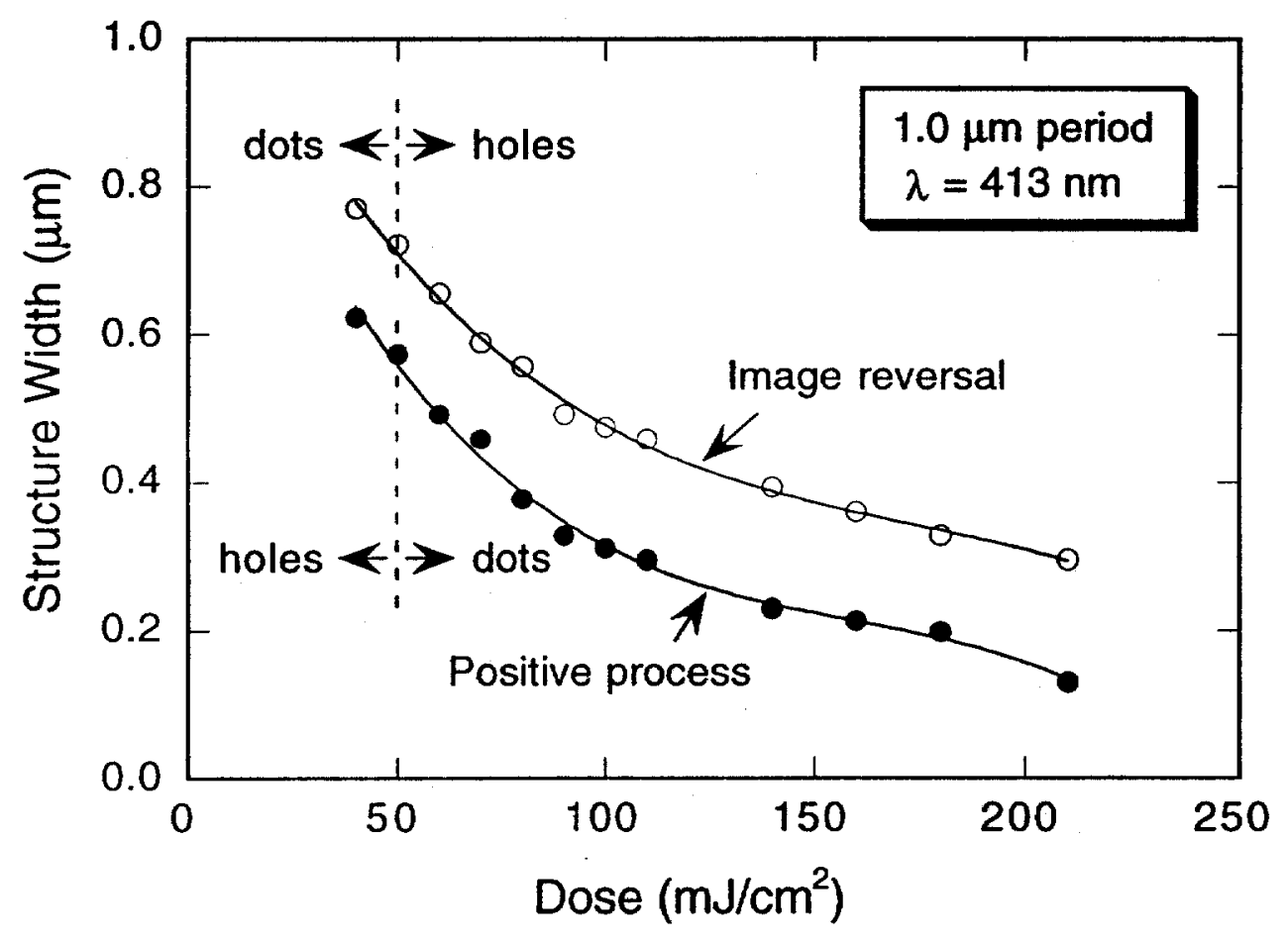

Figure 2 
A. Fernandez et. al

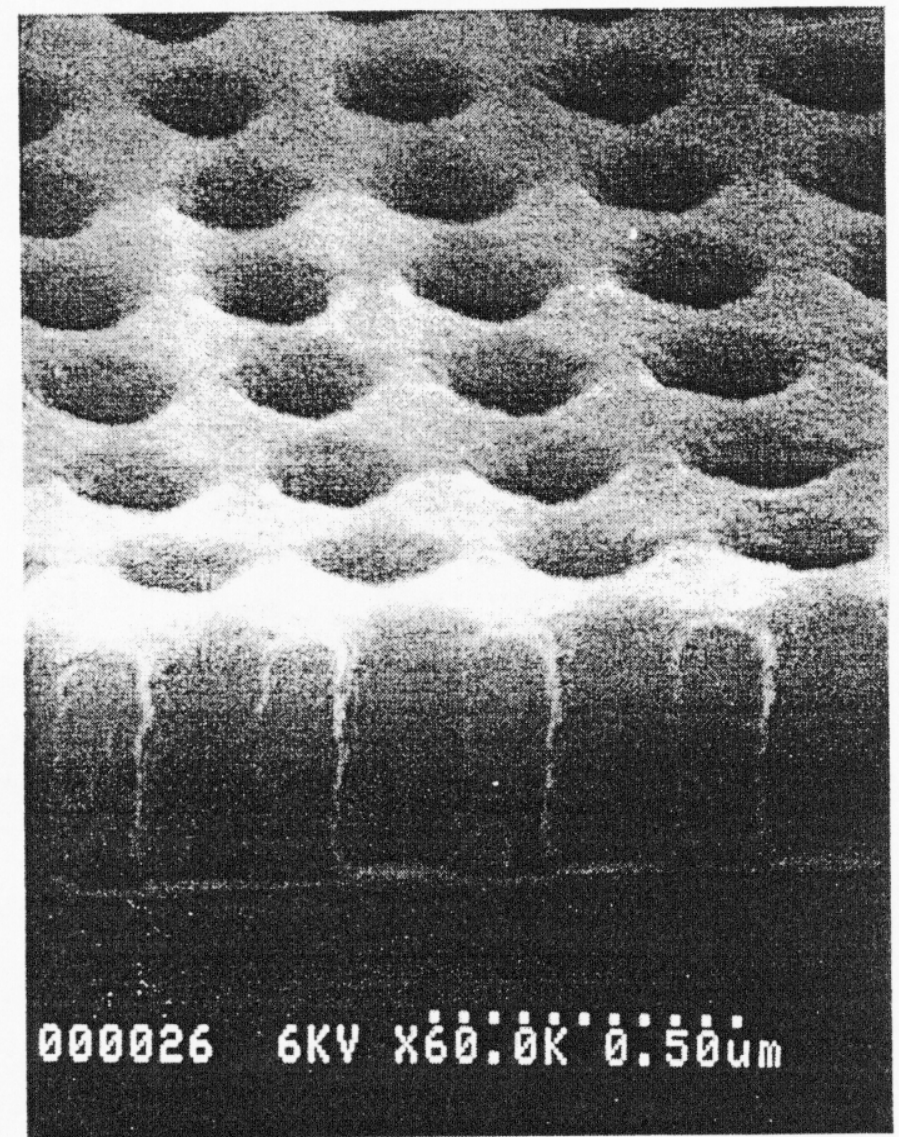

Figure 3 
A. Fernandez et. al
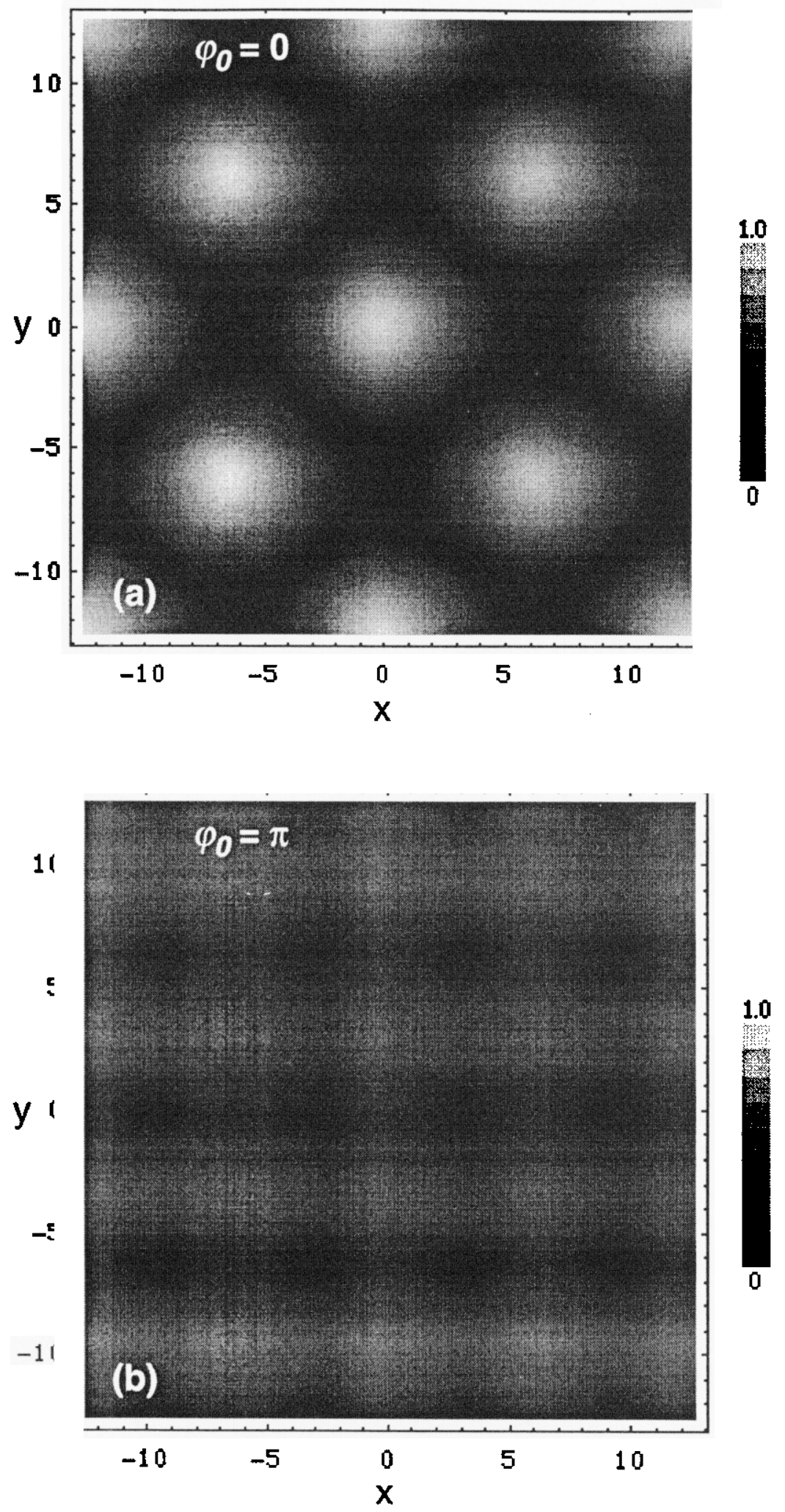

Figure 4 


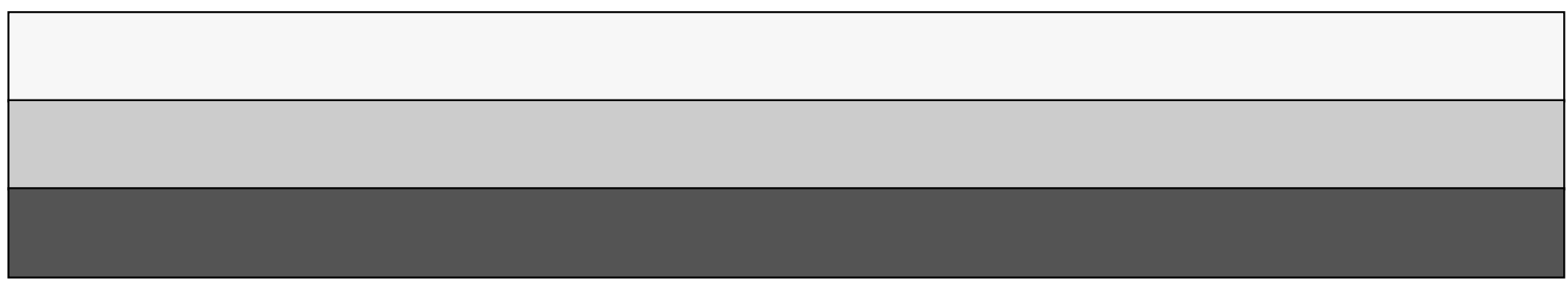

